\title{
Editorial
}

\section{Nonlinear Dynamics in Drilling Engineering}

\author{
Qilong Xue $\mathbb{D}^{1,2}$ Wenjun Huang $\mathbb{D}^{3},{ }^{3}$ Henry Leung, ${ }^{4}$ and Zhiqiao Wang $\mathbb{D}^{1,2}$ \\ ${ }^{1}$ School of Engineering and Technology, China University of Geosciences, Beijing 100083, China \\ ${ }^{2}$ Key Laboratory of Deep Geodrilling Technology, Ministry of Land and Resources, Beijing 100083, China \\ ${ }^{3}$ MOE Key Laboratory of Petroleum Engineering, China University of Petroleum, Beijing 102249, China \\ ${ }^{4}$ Department of Electrical and Computer Engineering, University of Calgary, Calgary, Alberta, Canada \\ Correspondence should be addressed to Qilong Xue; xql@cugb.edu.cn
}

Received 13 October 2021; Accepted 13 October 2021; Published 31 October 2021

Copyright (c) 2021 Qilong Xue et al. This is an open access article distributed under the Creative Commons Attribution License, which permits unrestricted use, distribution, and reproduction in any medium, provided the original work is properly cited.

Over the past 70 years, an increasing number of researchers have investigated the drillstring dynamics to understand its root causes, modelling, evaluation, control, and applications. The application of drillstring dynamics can bring immeasurable economic benefits for the oil industry. Consequently, the research and investigation of drillstring dynamics is an important and interesting problem.

Understanding drillstring dynamics is not only an interesting mechanics problem but also has considerable impact and help for the oil industry. The analytical, numerical, and experimental study of vibration and shock of the bottom hole drilling tool, such as rotary steerable system and drill bit measurement while drilling system, are of particular interest. The paper entitled "Mathematical Modelling and Dynamic Analysis of an Offshore Drilling Riser" by Liao et al. proposed a dynamic model of an offshore drilling riser is developed based on the Hamilton principle. The developed dynamic model is transformed into a finite element model by introducing an approximate solution which chooses the Hermite cubic interpolation function of the bending beam element as the shape function. The riser system working in deeper water with a higher top tension ratio and a lower buoyant factor shows more controllable vibration and less lateral deflection. Zeng et al. in the paper "Simulation Study on Dynamics of Hydraulic Turbines Used in Drilling Engineering" provided a detailed turbine design and a design method of turbine blade shape. Using the CFD (computational fluid dynamics) method, based on the realizable $k-\varepsilon$ turbulence model and Euler multiphase flow model, the effects of different external loads, blade numbers, blade installation angles, and flow rates on the force condition of turbine and the influence of different solid contents, particle sizes, and densities on turbine performance were studied.

Mechanical automatic vertical drilling tools (MAVDT) have gradually gained attention as a drilling tool that can achieve active correction in harsh working environments such as high temperature and high pressure. Wang et al. in the paper "Dynamic Characteristics and Key Parameter Optimization of Mechanical Automatic Vertical Drilling Tools" analyzes the force on the gravity sensing mechanism based on the structural analysis of the mechanical automatic vertical drilling tool. Then, the general dynamic equation of the gravity sensing mechanism is established based on D'Alembert principle. The multibody dynamics is introduced, and numerical analysis is used to analyze the motion characteristics of the gravity sensing mechanism under different initial conditions. Bai et al. in the paper "Research on the Dynamics of Geological Drilling Rig against Drill Pipe Impact" investigated the hydraulic system of the power head of the automatic drilling rig and studied the dynamics of the damage of the power head of the drilling rig caused by the impact of the drill pipe by means of hydraulic valve torque limit and closed-loop control of the speed. The research results of this paper can provide theoretical reference and design basis for subsequent development of automated drilling rigs.

The laboratory experiment is an effective method for exploring the behavior of drillstring vibration and its characteristics under different engineering backgrounds. This special issue collects three original contributions that take the laboratory experiment as a main research technique. 
Li et al. in the paper "Study of Radial Vibration Impact on Friction and Torque of Rotary Drill String" investigated the radial vibration characteristics by changing the major to minor radius ratio of the ellipse. A self-developed experimental device was designed to test the performance of tools with reduced friction and torque. The drillstring torque with different penetration rates, rotation speeds, and ratios of the major radius to minor radius of the ellipse were systematically studied. Du et al. in the paper "Experimental Study on the Dynamic Rock-Breaking Performance of Pulsed Abrasive Jet Drilling Method" pointed out that developing highefficiency drilling methods based on new combined water jets is a good approach to promote the rate of penetration (ROP) in such tight deep reservoirs. A pulsed abrasive water jet drilling tool is designed, and its dynamic work principle is analyzed. In the paper "Analysis on the Dynamics of Flexible Drillstring under Different Weight on Bit and Rotary Speed," we investigated a random collision model of flexible drill string, the actual drill string model is simplified by similar principles, and dynamic simulation software is introduced for simulation analysis. At the same time, an indoor experimental platform was set up to observe the movement law of the simulated drill pipe at different drilling speed and weight on bit (WOB). The trajectory of the point on the axis of the drill pipe in the entire wellbore would be observed, which is verified with the simulation results.

Dynamics measurement and data processing is also an essential aspect, as high-frequency measurement technology has become an important means of drilling string dynamics research in recent years. Li et al. in the review article entitled "Research and Development of High Frequency Measurement Technologies Used for Nonlinear Dynamics of Drillstring" reviewed the state of the art of high frequency measurement technologies used for nonlinear dynamics of drillstring, and data acquisition tools with high frequency sample rates, and the data processing are introduced. Based on high frequency data, the progress of drilling dynamics is summarized, including new understandings of low frequency drillstring dynamics, high frequency torsional oscillations (HFTO), high frequency torsional axial oscillations, and new findings for the coupling of vibrations and motions. In the future, studying the dynamics of the drillstring through measurement data will become the main method.

Nonlinear Dynamics in Drilling Engineering is a very large research topic. Although only a small part of the research results, we believe that this special issue will be useful for researchers and practitioners working in the broad drilling engineering.

\section{Conflicts of Interest}

The guest editors declare that they have no conflicts of interest regarding the publication of this special issue.

\section{Acknowledgments}

The guest editors would like to express gratitude to all the authors for their interest in selecting this special issue as a venue for disseminating their scholarly work.

Qilong Xue

Wenjun Huang

Henry Leung

Zhiqiao Wang 